\title{
Calcitonin doubling time in medullary thyroid carcinoma after the detection of distant metastases keenly predicts patients' carcinoma death
}

\author{
Yasuhiro Ito $^{1)}$, Akira Miyauchi ${ }^{1)}$, Minoru Kihara ${ }^{1)}$, Takumi Kudo ${ }^{2)}$ and Akihiro Miya ${ }^{1)}$ \\ 1) Department of Surgery, Kuma Hospital, Kobe, Japan \\ 2) Department of Internal Medicine, Kuma Hospital, Kobe, Japan
}

\begin{abstract}
Therapy using tyrosine-kinase inhibitors (TKIs) is now available for recurring or advanced medullary thyroid carcinoma (MTC). Here we investigated the calcitonin doubling time (Ct-DT) of MTC patients with distant recurrence postoperatively and for those with distant metastasis at the initial surgery. Of the 13 patients, six died due to the MTC at 5-93 months after the detection of distant metastasis. Their Ct-DTs were $\leq 1.58$ years. The remaining seven patients have been alive for 73-123 months after the detection of metastasis, and their Ct-DTs were low at $-4,-2.25$ years and 9.1733.92 years. Similar results were obtained by analyzing the value of $1 / \mathrm{Ct}-\mathrm{DT}$ to avoid discontinuity in the DT values among the patients with increasing serum $\mathrm{Ct}$ values over time and those with decreasing $\mathrm{Ct}$ values over time. These findings suggest that it is appropriate to use TKIs only for patients with a short Ct-DT and a large 1/Ct-DT with a cutoff at around 1.5 years and $0.67 /$ year, respectively, even if they have distant metastases.
\end{abstract}

Key words: Medullary thyroid carcinoma, Calcitonin doubling time, Distant metastasis, Tyrosine-kinase inhibitor

MEDULLARY THYROID CARCINOMA (MTC) arises from the c-cells of the thyroid and is classified as sporadic or hereditary based on the RET gene mutations. To date, large tumor size, node metastases, and extrathyroid extension have been identified as static prognostic factors for MTC [1]. In 1984, Miyauchi et al. demonstrated that after surgery, the change in the level of serum calcitonin $(\mathrm{Ct})$ over time - a significant tumor marker of MTC - accurately predicts a patient's prognosis, and they showed that the $\mathrm{Ct}$ doubling time (Ct-DT) is an important prognostic marker of MTC [2]. Thereafter, Barbet et al. performed a multivariate analysis that revealed the possibility that the Ct-DT and $1 / \mathrm{Ct}-\mathrm{DT}$ are prognostic factors superior to clinical staging [3]. They calculated $1 / \mathrm{Ct}-\mathrm{DT}$ in order to solve the problem of discontinuation in DT values among patients with increasing serum $\mathrm{Ct}$ values over time and those with decreasing $\mathrm{Ct}$ values over time.

In the past, there were no effective systemic therapies for MTC. However, vandetanib, a tyrosine-kinase

Submitted Mar. 14, 2016; Accepted Apr. 11, 2016 as EJ16-0140 Released online in J-STAGE as advance publication Apr. 19, 2016

Correspondence to: Akira Miyauchi, Department of Surgery, Kuma Hospital, 8-2-35, Shimoyamate-dori, Chuo-ku, Kobe 650-0011, Japan. E-mail: miyauchi@kuma-h.or.jp

(c) The Japan Endocrine Society inhibitor (TKI), has recently been widely used for advanced or recurrent MTC worldwide [4]. In Japan, other TKIs such as lenvatinib can also be used and are covered under the national health insurance system. This is epoch-making because, unlike the scenario for differentiated carcinomas, radioactive iodine therapy and thyroid-stimulating hormone suppression are not effective for treating advanced or recurrent MTC.

However, the indications for TKIs as well as when and how they should be administered remain unclear. It is doubtful whether TKI administration is beneficial for all MTC patients with distant metastasis, because of the potential various adverse events. In this study, we investigated the relationship between the Ct-DT in MTCs showing distant recurrences after surgery or MTCs with distant metastases at surgery (M1) and the prognoses of the patients, in order to elucidate the indications for TKIs.

\section{Patients and Methods}

\section{Patients}

Between November 1969 and April 2014, 290 patients underwent surgery for MTC at Kuma Hospital. Of these, nine patients had distant metastasis at sur- 
gery (M1) and 12 showed distant recurrences, to date. In this study, we enrolled 13 patients (four males, nine females) who underwent surgery for M1 MTC (two patients) or showed distant recurrence (11 patients) between July 1991 and April 2014. One M1 patient was excluded because she was enrolled in a clinical trial of TKI soon after her surgery. The measurements of the enrolled patients' $\mathrm{Ct}$ were performed using the same assay, i.e., a double antibody immunoassay using the same reagent. Of the 13 patients, the case seven were sporadic and those of the other six were hereditary, which was confirmed by a RET mutation analysis. The patients' ages at the initial surgery and at the appearance of distant recurrence were 18-78 years and $37-78$ years, respectively. In 11 patients, we measured the serum $\mathrm{Ct}$ level at the first four time points or more after the detection of distant recurrence on imaging studies. For the two M1 patients, we measured the serum $\mathrm{Ct}$ level after thyroid surgery.

After recurrence, none of the patients were administered TKIs during the period of our investigation, since no TKIs were approved by the Japanese government at that time except for use in clinical trials. One patient underwent extrabeam radiotherapy (EBRT) for bone metastases after they were detected, and the $\mathrm{Ct}$ values were measured only after the EBRT was conducted. Ten patients also showed local recurrences in the remnant thyroid or regional lymph nodes, but these recurrences were treated by re-operation or not treated. In all cases, these lesions were stable.

\section{The Ct assay}

The Ct assay was performed by double antibody radioimmunoassay in all 13 patients as indicated above. We used the same assay method from September 1983 onward, but the test reagent was changed in July 1991. In April 2015, we started using an electro-chemiluminescence immunoassay (ECLIA) method for the $\mathrm{Ct}$ assay, but no patients treated after April 2015 were enrolled in this study.

\section{Measurement of serum Ct and calculation of the Ct-DT}

Normally, the frequency with which serum Ct was measured after surgery ranged from two to four times per year. In one patient, $\mathrm{Ct}$ could be measured at only time points after the detection of distant recurrence because the patient died 12 months after the distant recurrence. In this patient, we included two more $\mathrm{Ct}$ measurements obtained just before the detection of distant recurrence in order to calculate the Ct-DT. We measured $\mathrm{Ct}$ once per 2 months in another patient, who died only 8 months after distant recurrence. We calculated the Ct-DT of these patients using the Ct levels as indicated above. We used the Doubling Time and Progression Calculator provided on our homepage (www.kuma-h.or.jp/), which is based on the calculation method developed by Akira Miyauchi [2].

\section{Results}

Of the 13 patients, six (46\%) died of MTC from eight to 93 months after the detection of distant metastases (Table 1); all six patients died of the progression of metastatic lesions. The Ct-DTs of these six patients were short, ranging from 0.25 to 1.25 years for the first four time points and from 0.25 to 1.58 years for all points after the detection of distant metastases. Two patients had the RET gene mutations $790 \mathrm{Leu}>\mathrm{Phe}$ and $618 \mathrm{Cys}>\mathrm{Ser}$, which are regarded as being of moderate risk in the newest American Thyroid Association (ATA) guidelines [5]. Four patients were $>60$ years old when their distant recurrences appeared.

The basal Ct levels when distant metastases were found varied among the patients. Three of these levels were comparably low, at $320-1,100 \mathrm{pg} / \mathrm{mL}$, and the remaining three were high, at $26,000-66,000 \mathrm{pg} / \mathrm{mL}$. Two patients were M1, and in one patient distant recurrence was detected only 11 months after surgery. The periods between initial surgery and the detection of distant recurrence in the remaining three patients were comparably long, at 180-327 months. All patients died of MTC 8-93 months after the detection of distant recurrence.

Table 2 summarizes the profiles of the seven patients who have remained alive with distant metastases over periods ranging from 73 to 123 months. The $\mathrm{Ct}$ levels were stable in all of these patients, and their Ct-DTs were very low, ranging from -0.83 to -37.75 years for the first four time points, and $-4,-2.25$ years and from 9.08 to 33.92 years for all points. Four patients had the RET gene mutations $634 \mathrm{Cys}>\mathrm{Arg}$ (three patients) and $634 \mathrm{Cys}>\mathrm{Tyr}$ (one patient), which are high-risk mutations in the ATA guidelines [5].

All seven of the surviving patients underwent initial surgery and showed distant recurrences at ages $<60$ years. Their basal $\mathrm{Ct}$ levels at the appearance of distant recurrence were comparably low, at 1,800 and 2,600 
Table 1 Profiles of six patients who died with distant metastasis

\begin{tabular}{|c|c|c|c|c|c|c|c|c|c|c|}
\hline No. & Gender & RET mutation & $\begin{array}{l}\text { Age at initial } \\
\text { surgery } \\
(\text { yrs })\end{array}$ & $\begin{array}{l}\text { Age at distant } \\
\text { recurrence } \\
(\mathrm{yrs})\end{array}$ & $\begin{array}{l}\text { Organs of } \\
\text { recurrence }\end{array}$ & $\begin{array}{c}\text { Basal Ct* } \\
\text { (pg/mL) }\end{array}$ & $\begin{array}{c}\text { Ct-DT } \\
\text { (4 points) } \\
\text { (yrs) }\end{array}$ & $\begin{array}{c}\text { Ct-DT } \\
\text { (All points) } \\
\text { (yrs) }\end{array}$ & $\begin{array}{l}\text { Period until } \\
\text { recurrence } \\
\text { after surgery } \\
(\mathrm{mos})\end{array}$ & $\begin{array}{l}\text { Period until } \\
\text { death after } \\
\text { recurrence } \\
(\mathrm{mos})\end{array}$ \\
\hline 1 & $\mathrm{~F}$ & No & 62 & 76 & Liver & 28,000 & 0.5 & 0.13 & 180 & 89 \\
\hline 2 & M & 790Leu $>$ Phe & 67 & $67^{* *}$ & Bone & 26,000 & 0.92 & 1.58 & 0 & 93 \\
\hline 3 & $\mathrm{~F}$ & $618 \mathrm{Cys}>$ Ser & 37 & 66 & Liver, Bone & 66,000 & 1.25 & 1.25 & 327 & 16 \\
\hline 4 & $\mathrm{~F}$ & No & 37 & 38 & Liver, Bone, Lung & 650 & $0.25^{* * *}$ & $0.25^{* * *}$ & 11 & 12 \\
\hline 5 & $\mathrm{~F}$ & No & 78 & $78^{* *}$ & Lung & 320 & 0.25 & 0.25 & 0 & 8 \\
\hline 6 & $\mathrm{M}$ & No & 38 & 59 & Lung, Bone & 1,100 & 0.67 & 1.08 & 250 & 73 \\
\hline
\end{tabular}

${ }^{*}$ At the time of appearance of distant recurrence. ${ }^{* *}$ Distant metastases were observed at initial surgery. ${ }^{* * *}$ Two points before the appearance of metastasis were included.

Table 2 Profiles of seven patients who are alive with distant metastasis

\begin{tabular}{|c|c|c|c|c|c|c|c|c|c|c|}
\hline No. & Gender & RET mutation & $\begin{array}{l}\text { Age at initial } \\
\text { surgery } \\
\text { (yrs) }\end{array}$ & $\begin{array}{l}\text { Age at distant } \\
\text { recurrence } \\
(\mathrm{yrs})\end{array}$ & $\begin{array}{l}\text { Organs of } \\
\text { recurrence }\end{array}$ & $\begin{array}{c}\text { Basal Ct* } \\
(\mathrm{pg} / \mathrm{mL})\end{array}$ & $\begin{array}{c}\text { Ct-DT } \\
\text { (4 points) } \\
\text { (yrs) }\end{array}$ & $\begin{array}{c}\text { Ct-DT } \\
\text { (All points) } \\
\text { (yrs) }\end{array}$ & $\begin{array}{l}\text { Period until } \\
\text { recurrence } \\
\text { after surgery } \\
\text { (mos) }\end{array}$ & $\begin{array}{l}\text { Period of } \\
\text { living with } \\
\text { recurrence } \\
(\mathrm{mos})^{* *}\end{array}$ \\
\hline 1 & $\mathrm{~F}$ & $634 \mathrm{Cys}>\mathrm{Arg}$ & 18 & 57 & Liver & 2,600 & -37.75 & 11.01 & 467 & 86 \\
\hline 2 & $\mathrm{~F}$ & No & 25 & 59 & Lung & 100,000 & -0.83 & -4 & 401 & 106 \\
\hline 3 & M & No & 43 & 59 & Bone & 17,000 & -0.44 & -2.25 & 201 & 121 \\
\hline 4 & $\mathrm{~F}$ & No & 36 & 43 & Lung & 50,000 & -1.5 & 9.08 & 92 & 121 \\
\hline 5 & $\mathrm{~F}$ & 634Cys $>$ Tyr & 19 & 41 & Lung, Liver & 26,000 & -2 & 33.92 & 262 & 73 \\
\hline 6 & $\mathrm{~F}$ & $634 \mathrm{Cys}>\mathrm{Arg}$ & 27 & 48 & Lung & 78,000 & -21.50 & 9.17 & 136 & 123 \\
\hline 7 & M & $634 \mathrm{Cys}>\mathrm{Arg}$ & 53 & 54 & Bone & 1,800 & -21.58 & 14.67 & 21 & 110 \\
\hline
\end{tabular}

* At the time of appearance of distant recurrence. ${ }^{* *}$ All patients are still alive.

$\mathrm{pg} / \mathrm{mL}$ in two patients, but those in the remaining five patients were high, ranging from 17,000 to 100,000 $\mathrm{pg} / \mathrm{mL}$. It took more than 120 months after surgery until distant recurrence occurred in five of the seven patients. On imaging studies, the metastastic lesions gradually progressed in patient no. 4 , but those in the remaining six patients were stable during follow-up.

We next investigated the change in the inverse values of Ct-DT (1/Ct-DT) to avoid discontinuity of the measured values, because the Ct-DTs of the patients who had decreasing serum $\mathrm{Ct}$ values over time were given as negative values. As shown in Fig. 1, the value of $1 / \mathrm{Ct}$-DT at the first four time points (a) did not differ much from those of all points (b) in each patient. Patients who died from theirs MTCs had Ct-DTs $\geq 1.58$ years and $1 / \mathrm{Ct}-\mathrm{DT}$ values $\geq 0.63$. In contrast, the patients who are alive with distant metastases had Ct-DTs $\geq 9.08$ years or negative Ct-DTs and a $1 / \mathrm{Ct}-\mathrm{DT}$ value $\leq 0.11$. The cutoff values among these groups were around 1.5 years for Ct-DT and around 0.67 for 1/Ct-DT.

We compared the relationship between the Ct-DT and the image diagnosis of metastasis and found that

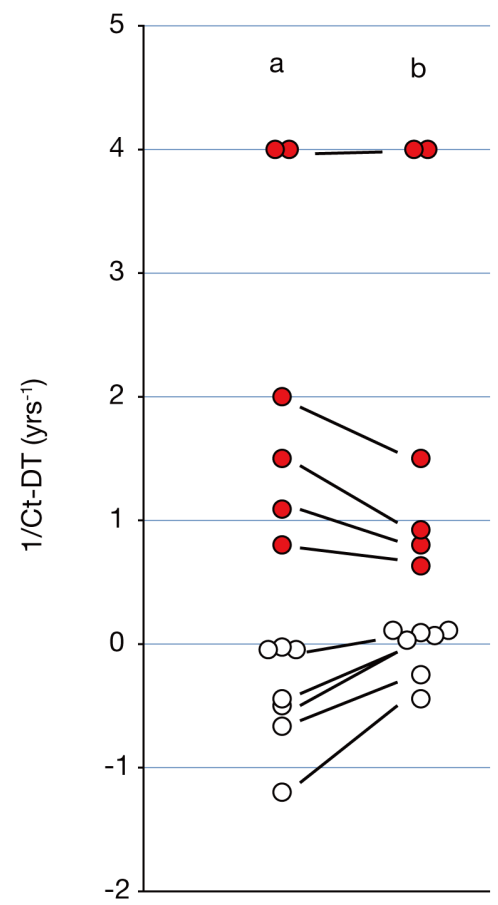

Fig. 1 Plot of 1/Ct-DT for four points (a) and all points (b) of 13 MTC patients. Patients with red dots died due to MTC, and those with white dots are still alive. 
the metastases of all patients with long Ct-DTs were stable. The patients with short Ct-DTs showed progressive metastases or newly appeared metastases, but we could not evaluate these lesions accurately because constant systemic imaging studies had not been performed in most of these cases.

\section{Discussion}

As we previously reported, the cause-specific survival (CSS) of MTC patients in Japan is good: the 5-year and 10-year CSS rates are $96.6 \%$ and $91.7 \%$, respectively [1]. The Ct-DT was first proposed by Akira Miyauchi to predict whether and when patients will die from MTC after surgery [2]. He and his colleagues showed that in their patient series, all of the patients with a Ct-DT $<6$ months died from the MTC within 3 years, whereas none of the patients with a Ct-DT $>6$ months died within 3 years.

Barbet et al. also showed that the Ct-DT was an independent prognostic factor of MTC [3]. They also proposed the 1/Ct-DT to avoid discontinuity in the values when the Ct-DT of any patient was $<0$. In these two studies, the serum $\mathrm{Ct}$ levels were obtained immediately after the initial surgery [3].

However, we often encounter the cases in which the Ct-DT suddenly becomes shorter because of the novel appearance or growing activity of metastatic lesions. The Ct-DT (and also 1/Ct-DT) immediately after surgery predict patients' prognoses, but these values are not always constant, especially when unresectable distant metastases appear or grow. In the past, no systemic therapies were available for MTC with distant recurrence or metastasis at surgery.

Some TKIs can be administered to patients with recurrent or advanced MTC [4]. However, these medicines are associated with various adverse events (AEs), which may significantly damage the patients' quality of life. The occurrence of distant metastasis or recurrence suggests that the MTC is very progressive and will have a dire prognosis. It may thus have been thought that TKIs should be administered to all MTC patients who exhibit distant metastases, because no other therapeutic strategies are available.

However, the results of the present study demonstrated that the CSS of MTC patients with distant metastases depends significantly on the Ct-DT after the appearance of distant metastases, because all of our patients whose Ct-DTs were $\leq 1.25$ years at the first four points died of carcinoma, whereas the $\mathrm{Ct}$ levels were stable for all of the patients who remain alive with distant metastases. Therefore, TKIs should be administered to patients with distant metastases whose Ct-DTs are short, but not to those with long Ct-DTs.

The number of patients in our series is too small for any statistical analyses to be performed, but we observed some differences in characteristics by comparing the patients who died due to their MTC with the patients who remain alive with metastases. The age at distant recurrence of the patients who died of MTC was higher (four of six patients were $>60$ years old) than that of the patients who remain alive with metastases (all were $<60$ years old). The periods until recurrence after surgery in the patients who died of MTC were shorter (two were M1 and one was 11 months) than those of the patients who remain alive with metastases (all were $>20$ months). These factors may statistically predict patients' prognoses after distant recurrence.

The risk classification of the RET mutations seems not to have affected the patients' prognoses after distant recurrence, because two of our six patients who died from their MTC had moderate-risk RET mutations, whereas four of the seven patients who are surviving with metastases show high-risk mutations according to the ATA guidelines [5].

In this study, the Ct-DT values of the patients who died due to MTC were $\leq 1.25$ years at the first four points, whereas those for the patients who survive were $<0$ at the first four points and $-4,-2.25$ and 9.08 years or longer. In addition, the values of 1/Ct-DT in all of the patients who died of MTC were higher than those of all of the patients who survive. However, previous studies showed that Ct-DT values range widely and are not polarized [1]. It remains unknown whether and why the Ct-DTs were clearly delineated between the high and low values in our series. Further studies of larger numbers of patients are necessary to elucidate this point. As shown in Fig. 1, there was no significant difference between the 1/Ct-DT values at the first four points and those at all points. We can thus predict the CSS of MTC patients by calculating their Ct-DT or 1/ Ct-DT at the first four points after the recurrence of distant metastases.

Another limitation of this study is that we could not accurately compare the Ct-DTs and the changes in metastatic lesions on imaging studies. Enlarged metastases or novel metastases to other organs appeared in patients with short Ct-DTs, but we could not accurately 
confirm the relationship. However, when the Ct-DT becomes short and existing metastases do not significantly change, it is better to search for novel metastases by fluorodeoxyglucose (FDG)-positron-emission tomography (PET) in order to identify target lesions of TKI therapy.

In summary, the Ct-DT and $1 / \mathrm{Ct}-\mathrm{DT}$ can be predic- tors of CSS in MTC patients who have distant metastases. Clinicians should thus regard MTC patients with distant recurrences as candidates for the administration of TKIs when their Ct-DTs are short or the $1 / \mathrm{Ct}$-DT is high. Cutoff values for considering the administration of the current TKIs might be around 1.5 years for the $\mathrm{Ct}-\mathrm{DT}$ and around $0.67 /$ year for 1/Ct-DT.

\section{References}

1. Ito Y, Miyauchi A, Yabuta T, Fukushiim M, Inoue H, et al. (2009) Alternative surgical strategies and favorable outcomes in patients with medullary thyroid carcinoma in Japan: experience of a single institution. World J Surg 33: 58-66.

2. Miyauchi A, Onishi T, Morimoto S, Takai S, Matsuzuka F, et al. (1984) Relation of doubling time of plasma calcitonin levels to prognosis and recurrence of medullary thyroid carcinoma. Ann Surg 199: 461-466.

3. Barbet J, Campion L, Kraeber-Bodere F, Chatal JF, GTE Study Group (2005) Prognostic impact of serum calcitonin and carcinoembryonic antigen doubling-time in patients with medullary thyroid carcinoma. J Clin Endocrinol Metab 90: 6077-6084.

4. Wells SA Jr, Robinson BR, Gagel RF, Dralle H, Fagin JA, et al. (2012) Vandetanib in patients with locally advanced or metastatic medullary thyroid cancer: a randomized, double-blind phase III trial. J Clin Oncol 30: 134-141.

5. Wells SA Jr, Asa SL, Dralle H, Elisei R, Evans DB, et al. (2015) Revised American Thyroid Association Guidelines for the management of medullary thyroid carcinoma. Thyroid 25: 567-610. 